Original Article

\title{
Influence of ankle dorsiflexion range of motion on heel-rise time during gait
}

\author{
Min-Hyeok Kang, PT, PhD ${ }^{1)}$ \\ 1) Department of Physical Therapy, International University of Korea: 965 Dongburo, Munsaneup, \\ Jinju, Gyeongsangnam-do 52833, Republic of Korea
}

\begin{abstract}
Purpose] The purpose of this study was to examine the correlation between weight-bearing (WB) and non-WB ankle dorsiflexion (DF) range of motion (ROM) and ankle movement during gait, including heel-rise time and ankle DF at heel-rise. [Subjects and Methods] Thirty healthy male subjects were recruited for this study. Ankle DF ROM of both feet was measured under the WB and non-WB conditions. Heel-rise time and ankle DF at heel-rise in both feet during gait were measured using a motion analysis system. Pearson product moment correlations were used to identify correlation ankle DF ROM and ankle movement during gait. [Results] Heel-rise time and ankle DF at heel rise were significantly correlated with WB ankle DF ROM. However, no correlations were found between ankle movement during gait and non-WB ankle DF ROM. [Conclusion] These findings demonstrate that WB ankle DF ROM measurements can be used to predict heel-rise time and ankle DF at heel-rise.

Key words: Ankle dorsiflexion, Gait, Heel-rise
\end{abstract}

(This article was submitted Jan. 2, 2018, and was accepted Feb. 19, 2018)

\section{INTRODUCTION}

Normal ankle movement is a crucial factor in preventing lower extremity injuries ${ }^{1}$. Abnormal or faulty ankle movements during gait are associated with ankle lower-extremity overuse injuries, plantar fasciitis, and ankle sprain ${ }^{1-3)}$. Especially, early heel-rise leads to increased duration of weight bearing on the forefoot during stance phase of gait ${ }^{1,4)}$. Also, insufficient tibial advancement over the foot together with early heel-rise leads to midtarsal dorsiflexion and subtalar joint pronation, which increases stress on the forefoot ${ }^{5}$. Therefore, the method of measurement predicting the heel-rise time is important for prevention of lower-extremity injuries.

Generally, passive measurement of non-weight-bearing (non-WB) ankle dorsiflexion (DF) range of motion (ROM) is performed to predict ankle movement clinically. However, it has been suggested that non-WB ankle DF ROM is inadequate for prediction functional ankle movements ${ }^{6,7)}$. In line with this opinion, a previous study showed that maximum ankle DF during gait has greater correlation with WB ankle DF ROM than non-WB ankle DF passive $\mathrm{ROM}^{8)}$. However, despite the difference in time for heel-rise (i.e., end point of mid-stance phase) and maximum ankle DF (i.e., terminal stance phase) during gait, the previous study only demonstrated the correlation between WB ankle DF ROM and maximum ankle DF during gait ${ }^{8)}$. Therefore, whether WB ankle DF ROM is correlated with heel-rise time needs to be determined to develop a measurement strategy for the prediction of the heel-rise time. Thus, the aim of the present study was to examine the correlation between WB and non-WB ankle DF ROM and heel-rise time during gait.

\section{SUBJECTS AND METHODS}

In total, 30 healthy subjects (16 males and 14 females; mean age, $21.8 \pm 1.7$ years; mean height, $170.6 \pm 8.2 \mathrm{~cm}$; mean

Corresponding author. Min-Hyeok Kang (E-mail: kmhyuk01@gmail.com)

(C2018 The Society of Physical Therapy Science. Published by IPEC Inc.

(c) (1) $($ This is an open-access article distributed under the terms of the Creative Commons Attribution Non-Commercial No Derivatives

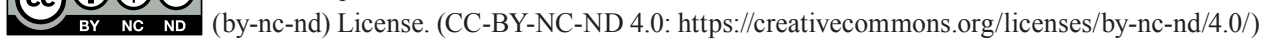


Table 1. Correlation between ankle dorsiflexion range of motion and ankle movement during gait

\begin{tabular}{lcc}
\hline \multicolumn{1}{c}{ Variables } & Heel-rise time & Ankle DF at heel-rise \\
\hline WB ankle DF ROM & $0.459^{*}$ & $0.303^{*}$ \\
Non-WB ankle DF ROM & 0.143 & 0.193 \\
\hline
\end{tabular}

$* \mathrm{p}<0.05$.

DF: dorsiflexion; ROM: range of motion; WB: weight-bearing.

Values are correlation coefficients $(r)$.

weight, $66.6 \pm 18.1 \mathrm{~kg}$ ) participated in this study. Potential subjects who had a history of lower-extremity surgery, ankle sprain during the last 1 year, or neurological disease were excluded. Prior to the experiment, all participants signed informed consent approved by the Institutional Research Review Committee of Inje University. The study protocol complies with the ethical standards of the Declaration of Helsinki.

WB and non-WB ankle DF ROM measurements were performed according to a previous study protocol ${ }^{9,10)}$. The WB ankle DF ROM was measured with subjects standing in front of the wall. The tested leg was placed behind the contralateral leg in a lunge posture ${ }^{9)}$. To allow natural subtalar joint pronation during weight bearing, the big toe and the middle of calcaneus were placed in a straight line ${ }^{11)}$. Subjects were instructed to place both hands on the wall and lean forward to attain the maximum tolerable stretch of the tested leg without knee flexion. An examiner measured tibial inclination using inclinometer upon reaching the maximum ankle $\mathrm{DF}^{9)}$. The non-WB ankle DF ROM with extended knee was measured passively using a goniometer in the prone position ${ }^{10)}$. An examiner applied force to the mid- and forefoot in the neutral subtalar joint position until it felt firm. The non-WB ankle DF passive ROM was measured at the firm end using a goniometer. Ankle DF ROM measurements were repeated three times under WB and non-WB conditions, respectively. To measure heel-rise time during gait, VICON motion analysis system (Vicon Motion Systems Ltd., Oxford, UK) with a sampling rate of $100 \mathrm{~Hz}$ was used. Reflective markers were attached to the lower-extremity according to the Plug-in-Gait model ${ }^{12)}$. Heel-rise time was determined when the marker on the lateral malleolus was elevated $4 \mathrm{~mm}$ from the height of the lateral malleolus marker in the flat-foot position during gait ${ }^{12)}$. Heel-rise time was normalized to the stance time of each subject. Ankle DF at heel-rise was calculated using relative angle between shank segment and ankle segment at heel-rise. All subjects performed gait trials three times. All measurements (e.g., non-WB ankle DF ROM, WB ankle DF ROM, and gait variables) were conducted on both feet.

For data analysis, the mean value of each three test trials was calculated. Pearson product moment correlations were used to correlate the ankle DF ROM with heel-rise time, and the ankle DF ROM with ankle DF at heel-rise. All statistical analysis were performed using PASW Statistics software (ver. 18.0; SPSS, Inc., Chicago, IL, USA) with an alpha level of 0.05.

\section{RESULTS}

WB ankle DF ROM was significantly correlated with heel-rise time $(p<0.001)$ and ankle DF at heel-rise $(p=0.019$; Table 1). However, non-WB ankle DF ROM was not significantly correlated with heel-rise time $(\mathrm{p}=0.277)$ and ankle $\mathrm{DF}$ at heel-rise $(\mathrm{p}=0.140)$.

\section{DISCUSSION}

The present study demonstrated that heel-off time is significantly correlated with WB ankle DF ROM (p<0.05) but not with non-WB ankle DF ROM ( $\mathrm{p}>0.05)$. Maximum ankle DF occurred in terminal stance during gait ${ }^{13)}$. However, because terminal stance phase is defined as the period from heel-rise to foot strike of the other foot, the area of weight support is transferred to the mid- and forefoot during terminal stance ${ }^{13)}$. Thus, it is more important to identify heel-rise time than maximum ankle DF to indirectly predict the stress on forefoot during gait. Nevertheless, in the previous study, only correlation between ankle DF ROM and maximum ankle DF during gait was reported ${ }^{8}$. Based on our findings, we demonstrated that ankle DF ROM measurement under the WB condition is an attractive option to predict heel-rise time. Considering that early heel-rise leads to greater stress on the forefoot and subsequently contributes to lower-extremity overuse injuries ${ }^{1)}$, the measurement method that indirectly predicts heel-rise time may be useful clinically.

Ankle DF at heel-rise was also significantly correlated with only WB ankle DF ROM in the present study $(\mathrm{p}<0.05)$. A previous study showed that the force applied to the foot was greater under WB than non-WB condition ${ }^{8}$. During mid-stance phase of gait, a relatively larger force is applied to the foot and ankle on the stance limb bearing the body weight ${ }^{13)}$. Thus, the ankle DF ROM measured under the WB condition may better reflect ankle movement during gait, which may influence our findings. Based on our findings, WB ankle DF ROM measurements can be used to predict heel-rise time and ankle DF at heel-rise in the clinical setting.

There are some limitations in the present study. First, only young individuals were recruited for this study. Thus, it is dif- 
ficult to generalize the results across different ages. Second, only ankle DF ROM was used to predict heel-rise time. Further studies are needed to include other variables such as foot and ankle alignments.

\section{Funding}

This work was supported by International University of Korea's research fund in 2018.

\section{Conflict of interest}

None.

\section{REFERENCES}

1) Donatelli RA, Wooden MJ: Biomechanical orthotics. In: The Biomechanics of the Foot and Ankle, 2nd ed. Philadelphia: FA Davis Company, 1996.

2) Willems TM, Witvrouw E, Delbaere K, et al.: Intrinsic risk factors for inversion ankle sprains in male subjects: a prospective study. Am J Sports Med, 2005, 33: 415-423. [Medline] [CrossRef]

3) Kibler WB, Goldberg C, Chandler TJ: Functional biomechanical deficits in running athletes with plantar fasciitis. Am J Sports Med, 1991, 19: 66-71. [Medline] [CrossRef]

4) Johanson MA, Cooksey A, Hillier C, et al.: Heel lifts and the stance phase of gait in subjects with limited ankle dorsiflexion. J Ath1 Train, 2006, 41: 159-165. [Medline]

5) Karas M, Hoy DJ: Compensatory midfoot dorsiflexion in the individual with heelcord tightness: implications for orthotic device designs. J Prosthet Orthot, 2002, 14: 82-93. [CrossRef]

6) Rabin A, Kozol Z: Weightbearing and nonweightbearing ankle dorsiflexion range of motion: are we measuring the same thing? J Am Podiatr Med Assoc, 2012, 102: 406-411. [Medline] [CrossRef]

7) Whitting JW, Steele JR, McGhee DE, et al.: Passive dorsiflexion stiffness is poorly correlated with passive dorsiflexion range of motion. J Sci Med Sport, 2013, 16: 157-161. [Medline] [CrossRef]

8) Kang MH, Oh JS: Relationship between weightbearing ankle dorsiflexion passive range of motion and ankle kinematics during gait. J Am Podiatr Med Assoc, 2017, 107: 39-45. [Medline] [CrossRef]

9) Munteanu SE, Strawhorn AB, Landorf KB, et al.: A weightbearing technique for the measurement of ankle joint dorsiflexion with the knee extended is reliable. J Sci Med Sport, 2009, 12: 54-59. [Medline] [CrossRef]

10) Kang MH, Oh JS, Kwon OY, et al.: Immediate combined effect of gastrocnemius stretching and sustained talocrural joint mobilization in individuals with limited ankle dorsiflexion: a randomized controlled trial. Man Ther, 2015, 20: 827-834. [Medline] [CrossRef]

11) Bennell KL, Talbot RC, Wajswelner H, et al.: Intra-rater and inter-rater reliability of a weight-bearing lunge measure of ankle dorsiflexion. Aust J Physiother, 1998, 44: 175-180. [Medline] [CrossRef]

12) Kang MH, Kim JW, Choung SD, et al.: Immediate effect of walking with talus-stabilizing taping on ankle kinematics in subjects with limited ankle dorsiflexion. Phys Ther Sport, 2014, 15: 156-161. [Medline] [CrossRef]

13) Perry J, Burnfield JM: Gait analysis: normal and pathological function, 2nd ed. Thorofare: SLACK Inc., 2010. 